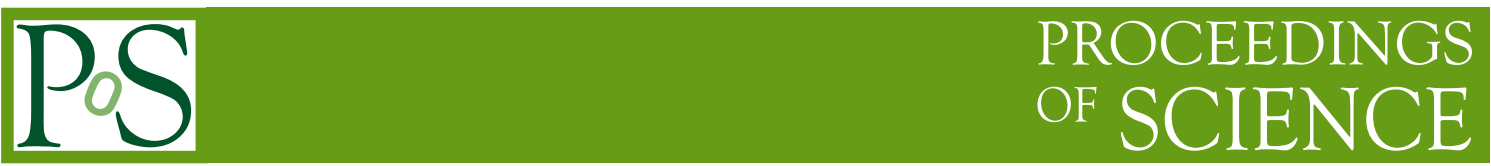

\title{
The INTEGRAL Galactic Plane Scanning
}

\author{
Mariateresa Fiocchi \\ Istituto di Astrofisica e Planetologia Spaziali. INAF. Italy \\ E-mail: mariateresa.fiocchi@iaps.inaf.it \\ Lorenzo Natalucci* \\ Istituto di Astrofisica e Planetologia Spaziali. INAF. Italy \\ E-mail: lorenzo.natalucci@iaps.inaf.it
}

\section{on behalf of the GPS Team}

\begin{abstract}
After the first nine years of INTEGRAL operational life, the discovery of new sources and source types, a large fraction of which are highly transient or highly absorbed, is certainly one of the most compelling results and legacies of INTEGRAL. Frequent monitoring of the Galactic Plane in AO8 and AO9 campaigns allowed us to detect transient sources, both known and new, confirming that the gamma-ray sky is dominated by the extreme variability of different classes of objects. Regular scans of the Galactic Plane by INTEGRAL provide the most sensitive hard X-ray wide survey to date of our Galaxy, with flux limits of the order of $0.3 \mathrm{mCrab}$ for an exposure time of $\sim 2 \mathrm{Ms}$. Many transient sources have been detected on a wide range of time scales ( $\sim$ hours to months) and identified by triggered followup observations, mainly by Swift/XRT and optical/infrared telescopes. These discoveries are very important to characterize the X-ray binary population in our Galaxy, that is necessary input for evolution studies. The transient source monitoring is crucial to sample a variety of physical conditions corresponding to a large span in luminosity. The long exposure times available allow us to define the spectral and timing properties of the known sources and the global properties of each object class.
\end{abstract}

"An INTEGRAL view of the high-energy sky (the first 10 years)" 9th INTEGRAL Workshop and celebration of the 10th anniversary of the launch,

October 15-19, 2012

Bibliotheque Nationale de France, Paris, France

\footnotetext{
*Speaker.
} 


\section{The INTEGRAL Galactic Plane Scanning}

For the first 5 years of INTEGRAL operational life, the scientific Core Programme included regular scans of the Galactic Plane and Bulge. These observations led to the discovery of many new high energy sources. Many of these are the highly absorbed HMXB, that were previously unknown due to the lack of sensitive hard X-ray observations. Furthermore, many new transient objects have been found, on short timescales and with low luminosity $\left(<10^{36}\right.$ ergs $\left.^{-1}\right)$. From AO-5 onwards, these regular scans were discontinued, resulting in a significant drop in the discovery rate of new Galactic systems. From AO8 to AO10 however, a multi-year proposal for Galactic Plane Scanning has been approved and since 2011 this program is collecting data for a total exposure time of $\sim 5 \mathrm{Msec}$ (at the end of 2013) to be added to the existing previous one, giving a total of $\sim 135$ Ms. The majority of these sources are best detected on timescales less than or equal to an INTEGRAL orbit ( $\sim 250 \mathrm{ks})$, so that scans occur once every revolution $(\sim 3$ days), increasing the chances to detect short outbursts from unknown and known sources and the possibility to monitor the evolution of long outbursts from different source classes. The observed sky region cover a strip of width \pm 10 degree in galactic latitude and a longitude extent of about $170 \mathrm{deg}$. Due to its unique capability to monitor the Galactic Plane for long periods at a sensitivity level unreachable by other wide-field imagers $\left(<\sim 0.3-0.5 \mathrm{mCrab}\right.$ for $\left.\mathrm{T}_{\text {exposure }}=1 \mathrm{Ms}, \sim 3 \mathrm{mCrab}_{\text {for }} \mathrm{T}_{\text {exposure }}=10 \mathrm{ks}\right)$, INTEGRAL has proven especially capable of discovering faint and/or short-lived phenomena that are typical of Galactic binary sources.
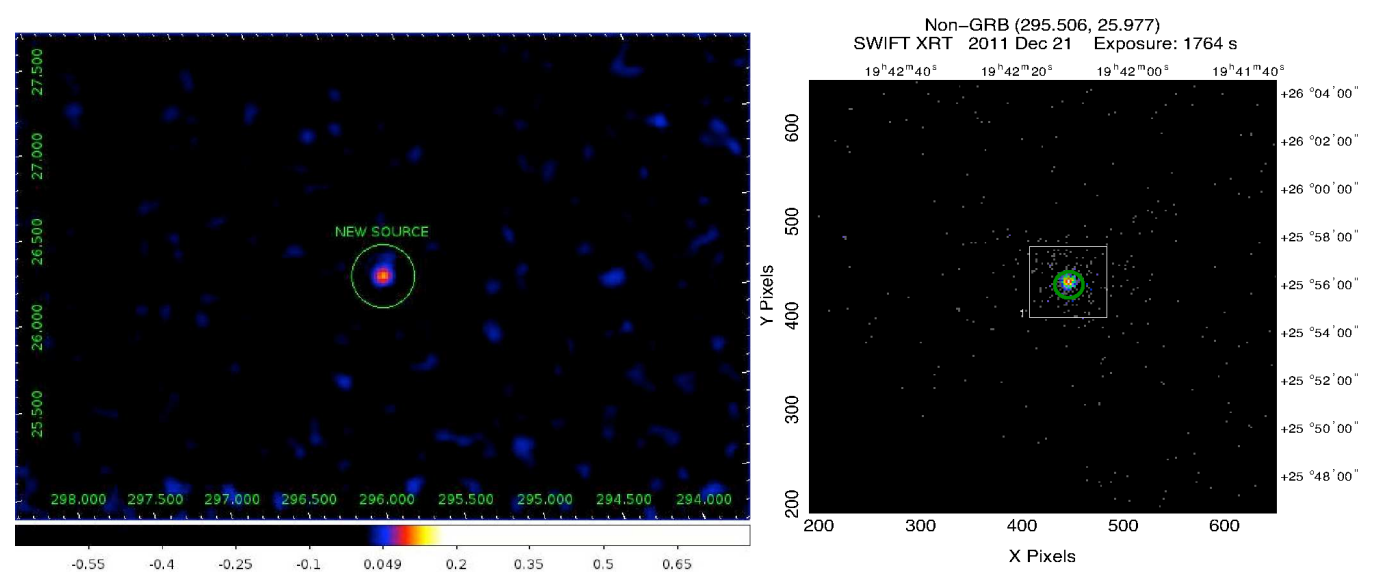

Figure 1: Left: JEM-X image in the 3-10 keV image of 1RXS J194211.9+255552 . Right: The XRT/Swift image in the 2-10 kev of the 1RXS J194211.9+255552

The Galactic Plane observations with INTEGRAL so far have changed our view of the Galaxy doubling the number of known high-mass X-ray binaries (Bird et al. 2010), discovering two new types of High Mass X-ray Binary (HMXB): 1) the high absorbed binary systems with $\mathrm{N}_{H} \geq$ $10^{24} \mathrm{~cm}^{-2}$, such that these sources could not be identified earlier with soft X-ray instruments (Kuulkers 2005; Ibarra et al. 2007); 2) the Super Giant Fast X-ray Transient (SFXT), showing the short (from tens of minutes to few hours) and intense flares $\left(\mathrm{L} \sim 10^{36-37}\right.$ ergs $\left.^{-1}\right)$, X-ray pulsar like spectrum, with very high dynamic range: 3-5 orders of magnitude with respect to the quiescent emission with $L \sim 10^{32} \mathrm{ergs}^{-1}$ (for review see Sidoli 2010, Sidoli 2011). The physics of these pro- 


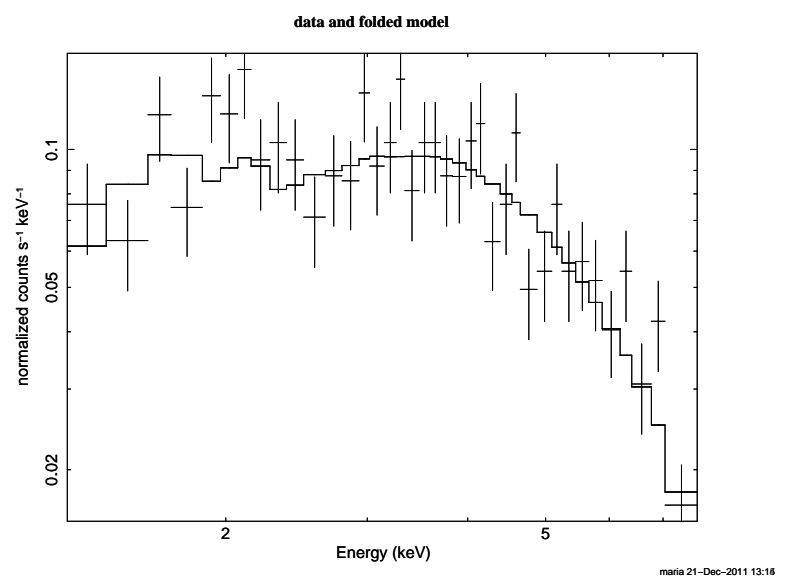

Figure 2: The XRT spectrum of ROSAT source 1RXS J194211.9+255552, fitted with a power law model with an absorption of $\mathrm{N}_{H} \sim 1.3 \times 10^{22} \mathrm{~cm}^{-2}$ and a photon index of $0.64 \pm 0.3$

cesses is still unknown and represent one of the main open issues in the study of compact objects in binary system. Studies of these HMXBs classes will be fundamental to understand the population synthesis, to determine the chemical enrichment of the Galaxy and the evolution of massive stars in binaries and to investigate the poorly understood accretion mechanism (Sidoli 2009 for a review) which produces the short flares observed from the SFXT.

The gamma-ray satellites Fermi and AGILE and the Cherenkov telescopes (as HESS, Milagro etc.) have provided new surveys of the Galactic Plane from $\mathrm{MeV}$ to $\mathrm{TeV}$ energies. INTEGRAL is and will be the unique opportunity to identifying counterparts of the unidentified very high energy sources and observations above $20 \mathrm{keV}$ are needed to understand the physical origin of their emission. In the second Fermi/LAT catalogue about $320 \mathrm{MeV} / \mathrm{GeV}$ sources have been reported within 10 degrees of the Galactic Plane and about one third are still unidentified (Nolan et al. 2013). Among them, some are transient sources flaring on timescales shorter than a few days, which can be a new class of the galactic fast transient high-energy emitters. INTEGRAL represent a good possibility to systematically identify the unidentified $\mathrm{MeV} / \mathrm{GeV} / \mathrm{TeV}$ sources, define the spatial distributions and luminosity functions of the each object classes.

The INTEGRAL observatory strategy and its large field of view allows to monitoring a large number and variety of galactic sources for a long time, needed to study the physics of emission mechanisms for white dwarfs, neutron stars and black holes. These observations allowed us to understand the interplay between the different components of the accretion flow onto the compact objects such as the cold disk and the neutron star, the hot Comptonizing plasma and the possible synchrotron radiating jets (Cadolle Bel et al., 2009; Prat et al., 2009; Fiocchi et al. 2008).// In particular we report here some INTEGRAL result: 1) non-thermal components at energies $>100$ $\mathrm{keV}$ from neutron star low-mass X-ray binaries (Fiocchi et al. 2006; Paizis et al. 2009; Tarana et al. 2011); 2) INTEGRAL discovered high energy emission (200-300 keV) in a strong magnetized neutron stars, with $B=10^{14-15}$ Gauss (Mereghetti et al. 2009 and and references therein) and the Soft Gamma Repeaters spectral behavior, soft at energies above $10 \mathrm{keV}$ and hard below $10 \mathrm{keV}$, in contrast to spectra from Anomalous X-ray Pulsars which show an opposite spectral shape; 3) hard emission from black holes up to $1 \mathrm{MeV}$ (Caballero Garcia et al., 2007; Del Santo et al. 2012). 


\section{The Galactic Plane Scanning throughout AO8 and AO9}

We briefly report on the preliminary results from the recent AO8 and AO9 Programme, concerning the discovery of the new source, monitoring of the know source and the timing study on long time scale. The results reported here are simple examples of the many different topics that can be studied with Galactic Plane observations.

\subsection{The new detections}

At the time of writing, the pointings of the Vela and Cygnus region starting in May 2011, allowed us to monitor many known sources: 31 HMXBs, 36 LMXBs, 5 AGN and 4 other sources (Atels 3361, 3434, 3816, 3818, 3887, 3916, 4135, 4136, 4168, 4218). The Galactic Plane Scanning observations (from AO1 to AO9) and the follow-up soft X-ray and optical/IR allowed to identify new sources in the Galactic plane: $\sim 50$ AGN hidden behind the galactic plane, $\sim 50$ HMXB, $\sim 20$ LMXB (Low Mass X-ray Binary) and $\sim 20 \mathrm{CV}$ (Cataclism Variables). Other $\sim 120$ new IGR sources have been found but their highly variable behavior makes it difficult to identify them, confirming that the gamma-ray sky is dominated by the extreme variability of different classes of objects (Bird et al. 2010).

A recent example of detection of a new source by INTEGRAL/JEM-X happened during the Galactic Plane Scan observations performed between 2011 December 18, 13:47 UTC and December 19, 8:59 UTC. This source has been promptly identified as the transient ROSAT 1RXS $\mathrm{J} 194211.9+255552$, with averaged fluxes of $10 \pm 2 \mathrm{mCrab}$ and $16 \pm 5 \mathrm{mCrab}$ in the $3-10 \mathrm{keV}$ and 10-25 keV energy ranges, respectively (Atel 3816). A Swift ToO follow-up has been performed on December 21, 2011 at 06:10:09.7 UTC, with a net exposure of $1756 \mathrm{~s}$. Within the ROSAT error circle only one source is found at position 19h42m11.13s, +25:56:07.32 (J2000), with 3.6 arcsec error radius (Atel 3818). Figure 1 shows the JEM-X image in the 3-10 keV energy range (left) and the XRT image in the $2-10 \mathrm{keV}$ energy range (right), while in Figure 2 the XRT spectrum of 1RXS J194211.9+255552 is shown. The best fit consisting of a simple absorbed power law model with an absorption of $\mathrm{N}_{H}=(1.3 \pm 0.6) \times 10^{22} \mathrm{~cm}^{-2}$, a photon index of $0.7 \pm 0.3$ and an unabsorbed flux of $\sim 7.9 \times 10^{-11} \mathrm{ergcm}^{-2} \mathrm{~s}^{-1}$, in 1-10 keV energy range (reduced $\chi^{2}=1.1$ for 36 degrees of freedom). Masetti et al. (Atel 4209) identified the optical counterpart using the $1.5 \mathrm{~m}$ Cassini telescope of the Astronomical Observatory of Bologna in Loiano (Italy) and showed that the optical counterpart is a Galactic high mass X-ray binary, confirming the HMXB nature for this source as suggested by ATel 3818.

\subsection{The monitoring of known sources}

During the Galactic Plane Scanning INTEGRAL perform a long monitoring of GS 0834-43, a source discovered by Granat/WATCH in February 1990 with a peak flux of $\sim 400$ mCrab in the 8-20 keV energy range (LapShov et al. 1992) and also observed at high energies by CGRO/BATSE at regular intervals until June 1993 (Wilson et al. 1997). These observations allowed to characterize GS 0834-43 as a Be transient X-ray pulsar with a spin period of $\sim 12 \mathrm{~s}$, a wide orbit (period of $\sim 106$ days), a small eccentricity between 0.10 and 0.17 and a low inclination angle (Wilson et al. 1997). After 1993, no outbursts were detected by BATSE or other monitoring instruments such as Swift/BAT (Cusumano et al. 2010) or INTEGRAL/IBIS (Bird et al. 2010). During INTEGRAL 

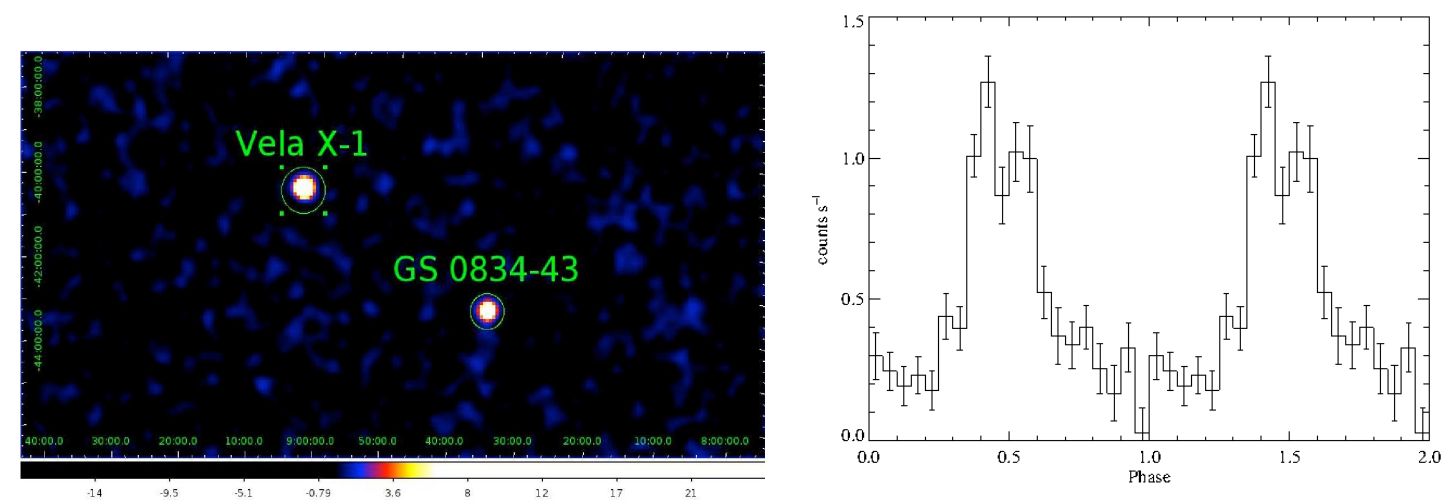

Figure 3: Left: IBIS image in the $18-60 \mathrm{keV}$ image during revolution 1184 of GS 0834-43. Right: The IBIS/ISGRI $18-60 \mathrm{keV}$ phase folded light curve using the best orbital period determination of 10.068 days of IGR J16328-4727

Galactic Plane Scan observations between 2012-06-26 06:44:07 and 2012-06-26 15:27:19 UTC, IBIS/ISGRI detected this source at 47 sigma confidence level in the 18-60 keV energy range with a exposure time of $15.8 \mathrm{ks}$. It was also detected by the JEM-X instrument at a significance of 19 sigma for an exposure time of $1.7 \mathrm{ks}$. The flux is $109 \pm 2 \mathrm{mCrab}$ in $18-60 \mathrm{keV}$ and $64 \pm 4 \mathrm{mCrab}$ in the $3-10 \mathrm{keV}$ energy range (Atel 4218). In Figure 3 (left) is shown the IBIS image of this field in the $18-60 \mathrm{keV}$ energy range. This new, strong detection signature of a renewed period of activity after a long period of quiescence was also detected with FERMI/GRBM (Atel 4235). Throughout the duration of the observations, the IBIS light curve in the 18-60 keV energy range shows that the source flux remained constant. The IBIS spectrum was well fitted with a simple power-law model with a photon index of $3.0 \pm 0.2$ and a flux of $1.8 \times 10^{-9} \mathrm{ergcm}^{-2} \mathrm{~s}^{-1}\left(\chi^{2}=12 / 9 \mathrm{dof}\right)$. The INTEGRAL detection of this new, strong outburst could be a signature of a renewed long term activity period that is being followed up by other orbiting high energy telescopes, also covering the hard X-rays. The Nuclear Spectroscopic Telescope Array (Nustar) has also observed this source in July 2012 with two pointings and is expected to provide better sensitive spectral/timing measurements.

\subsection{The timing studies}

The long exposure time reached in the Galactic Plane is an unique opportunity to perform timing studies, in particular to measure the orbital period of the X-ray binaries and to determine the spectral characteristics of the Galactic source. The long term 18-60keV IBIS/ISGRI light curve of IGR J16328-4726 is a very meaningful example of the detection of a period at $\sim 10$ days, which we interpret as the orbital period (for details see Fiocchi et al. 2012). The folded light curve using the best orbital period determination of $\sim 10$ days is shown in Figure 3 (right), where is detected a single broad emission maximum above a quiescent level with the shape similar to those observed from other SFXTs such as IGR J17544-2619 (Clark et al. 2009) and IGR J16465-4507 (Clark et al. 2010).

\section{Conclusions}

Thanks to the combination of its large field of view and good sensitivity for short exposures 
(20 mCrab is reached at $50 \mathrm{keV}$ with 2 standard GPS pointings of $2 \mathrm{ksec}$ each), timing resolution better than $1 \mathrm{~ms}$ and good broad band spectroscopy, INTEGRAL is and will be the only possibility to study very relevant topics as the new sources, the periodicity of Galactic sources, the spectral and temporal characteristic of the sources and of the different classes, their spatial distributions within our Galaxy and luminosity functions. Because of the arc-minute location accuracy at energies above $20 \mathrm{keV}$, INTEGRAL is and will be unique to find the hard-X counterparts of new unidentified $\mathrm{MeV} / \mathrm{GeV} / \mathrm{TeV}$ sources, discovered with AGILE and Fermi or with the ground based Cerenkov telescopes. Finally its instruments, sensitive in the $3 \mathrm{keV}$ to $10 \mathrm{MeV}$ range, provide a unique link between the soft X-ray band covered by X-ray telescopes such as XMM-Newton or Chandra and the energy band of the high-energy gamma-ray space missions. The future Galactic Plane observations will provide a more uniform and longer monitoring of the known sources, new source detections and will also detect outburst or in peculiar spectral states, triggering dedicated ToO.

\section{References}

[1] Bird et al. 2010, ApJS, 186,1

[2] Caballero Garcia et al., 2007, ApJ 669, 534

[3] Cadolle Bel et al., 2009, A\&A 501, 1

[4] Clark et al., 2009, MNRAS, 399, 113

[5] Clark et al., 2010, MNRAS, 406, 75

[6] Cusumano et al. 2010, A\&A, 510, 48

[7] Del Santo et al. 2013, MNRAS, 430, 209

[8] Fiocchi et al. 2006, ApJ, 651, 416

[9] Fiocchi et al. 2008, A\&A,492,557

[10] Fiocchi et al. 2012, ApJ, 762, 19

[11] Kuulkers 2005, AIPC News 797, 402

[12] Ibarra et al., 2007, A\&A ,465, 501

[13] LapShov et al. 1995, Soviet Astronomy Letters, Vol. 18, p. 12

[14] Mereghetti et al. 2009, ApJ 696, L74

[15] Nolan P.L. et al., 2012, ApJS, 199, 31

[16] Paizis et al. 2009, PASJ, 61, 107

[17] Prat et al., 2009, A\&A 494, L21

[18] Sidoli 2009, Advances in Space Research, 43, 1464

[19] Sidoli 2010, Symposium E, session 16, paper number E16-0026-10, COSPAR 38.2556S

[20] Sidoli et al. 2011, astro-ph/1111.5747

[21] Tarana et al. 2011, MNRAS, 416, 873

[22] Wilson et al. 1997, ApJ, 479, 388 